\title{
Study of Electronic Transport in Tunneling Devices Using an Incoherent Superposition of Time Dependent Wave Packets
}

\author{
XAVIER ORIOLS* and JORDI SUÑÉ \\ Departament d'Enginyeria Electrònica (ETSE), Universitat Autònoma de Barcelona, \\ 08193- Bellaterra, Spain
}

\begin{abstract}
A quantum beam of conserved particles is described as a Stationary Incoherent Superposition of Wave packets (SISOW). Mean values of a SISOW are time-independent confirming that the SISOW describes a stationary flux of particles. The SISOW is a general entity that contains the Hamiltonian eigenstates as a particular limit. The decomposition of the SISOW into wave packets provides intrinsic dynamic information which is not accessible from Hamiltonian eigenstates. Within the SISOW approach an exhaustive framework can be developed to be able to study dynamic phenomena in quantum electronic devices such us current fluctuations, relaxation and transient phenomena.
\end{abstract}

Keywords: Quantum devices; Tunneling; Mesoscopic physics; Electronic transport; Bohm trajectories; Shot noise

\section{INTRODUCTION}

The study of quantum systems in terms of wave packets has had little practical use for a long time because their experimental analysis seemed impossible. Among the various approaches used to study open systems, the one proposed by Landauer and Büttiker [1] has become a standard to treat coherent transport in mesoscopic devices. The Hamiltonian eigenstates are the main components of this approach since the conductance of the system is assumed to be proportional to their transmission coefficient. However, new advances in laser interaction with matter have brought the wave packet dynamics into the limelight to study ultrafast processes occurring on a time scale of femtoseconds [2]. In this paper we present an new approach to study electronic transport in quantum devices in terms of wave packets and Bohm trajectories. First of all, we will describe an original entity, based on the density matrix, that describes a beam of electrons as a constant flux of wave packets.

*Corresponding author. Tel.: (34-3) 58113 61, Fax: (34-3) 58131 50, e-mail: xavier.oriols@uab.es 


\section{STATIONARY INCOHERENT SUPERPOSITION OF WAVE PACKETS (SISOW)}

We are interested in describing the stationary flux of electrons impinging upon a localized potential, keeping at the same time the possibility of extracting consistent information about the dynamics of the interaction. We will study a one dimensional device located between two reservoirs, considering the injection of electrons from the left reservoir (the emitter). A time dependent wave packet, $\Phi_{\mathrm{t}_{\mathrm{o}}}(\mathrm{x}, \mathrm{t})$, can be written as a linear superposition of Hamiltonian eigenfunctions $\varphi_{E}(\mathrm{x})=$ $\langle\mathrm{x} \mid \mathrm{E}\rangle$ as:

$$
\begin{aligned}
\Psi_{t_{o}}(x, t) & =\left\langle x \mid \Psi_{t_{o}}(t)\right\rangle \\
& =\int_{0}^{+\infty} d E \cdot a(E) \cdot \varphi_{E}(x) \cdot e^{-i\left(E \cdot\left(t-t_{o}\right) /(\hbar)\right.}
\end{aligned}
$$

$a(E)$ being a complex quantity. From an intuitive point of view, a beam of particles can be thought as a constant flux of identical time-dependent wave packets each one leaving the emitter reservoir and entering into the device at different times $t_{0}$. Following this idea, we propose a description of a beam of electrons as an incoherent superposition of identical wave packets that enter into the system at different times with uniform probability. For such an incoherent superposition, we follow a formulation based on the density matrix, which in the energy representation is given by:

$$
\begin{aligned}
\rho_{\Psi}\left(E, E^{\prime}\right) & =\frac{1}{2 \pi h} \int_{-\infty}^{+\infty} d t_{o} \cdot\left\langle E \mid \Psi_{t_{o}}(t)\right\rangle\left\langle\Psi_{t_{o}}(t) \mid E^{\prime}\right\rangle \\
& =\frac{1}{2 \pi h} \int_{-\infty}^{+\infty} d t_{o} \cdot a(E) \cdot a^{*}\left(E^{\prime}\right) \cdot e^{-i\left(E-E^{\prime}\right)\left(t-t_{o}\right) /(h)}
\end{aligned}
$$

The constant $1 / 2 \pi \hbar$ comes from normalization in the sense that the trace is equal to unity. Expression (2) is the density matrix of a quantum entity that we will call a SISOW (Stationary Incoherent Superposition Of Wave packets).
Noting that:

$$
\int d t_{o} \cdot \exp \left(i\left(E^{\prime}-E\right) t_{o} / \hbar\right)=2 \pi \hbar \cdot \delta\left(E^{\prime}-E\right)
$$

it immediately follows that the final expression for the density matrix of a SISOW is:

$$
\rho_{\Psi}\left(E, E^{\prime}\right)=|a(E)|^{2} \delta\left(E^{\prime}-E\right)
$$

Thus, even though the density matrix associated to time-dependent wave packets is not diagonal, the density matrix of a SISOW is indeed diagonal. The mean value of an observable is given by:

$$
\langle\hat{A}\rangle=\operatorname{tr}(\hat{\rho} \cdot \hat{A})=\int_{0}^{\infty} d E \cdot|a(E)|^{2} \cdot A(E, E)
$$

where $\mathrm{A}(\mathrm{E}, \mathrm{E})$ are the diagonal elements of $\hat{A}$ in the energy representation. In particular, we notice that $\langle\hat{A}\rangle$ does not depend on time and this confirm that the SISOW is an adequate entity to describe stationary situations. As an example, the charge of a SISOW is time independent. The current density is time-independent, uniform, and proportional to the transmission coefficient of the wave packet. In general, we can say that the SISOW is a more flexible entity that contains the energy eigenstate as a monochromatic limit. In other words, an eigenstate can be considered as a SISOW with $a(E)=\delta\left(E-E^{\prime}\right) \quad$ (i.e., with an infinite spatial dispersion).

However, the main advantage of a SISOW resides on the dynamic information contained in its definition. In order to deepen into these dynamics information of a SISOW, let us make use of the method of quantum trajectories proposed by de Broglie and Bohm [3]. In addition to reproducing the standard quantum mechanical measurable results, the Bohm trajectories provide a valuable intuitive insight of the underlying physical processes. Within the Broglie-Bohm formalism, the velocity at each time and position, $\mathrm{v}_{\mathrm{t}_{\mathrm{o}}}(\mathrm{x}, \mathrm{t})$ is computed as:

$$
v_{t_{o}}(x, t)=J_{t_{o}}(x, t) /\left|\Psi_{t_{o}}(x, t)\right|^{2}
$$




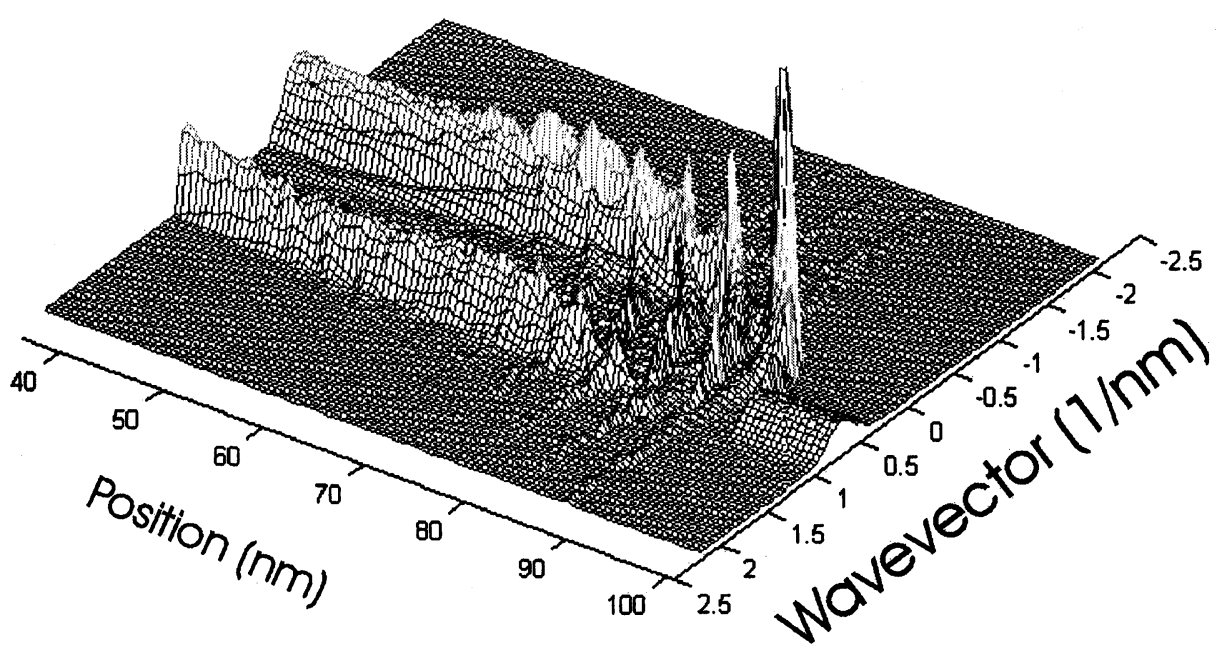

FIGURE 1 Phase space distribution for a single SISOW for a beam of electrons with central energy $0.015 \mathrm{eV}$ impinging upon a rectangular barrier of $20 \AA$ width and $0.04 \mathrm{eV}$ height. The behaviour before the barrier can be understood as a consequence of the current uniformity of the SISOW.

where $\mathrm{Jt}_{\mathrm{o}}(\mathrm{x}, \mathrm{t})$ is the current density associated to the wave packet. Let us now discuss the dynamic properties of a SISOW by evaluating the velocity. It can be straightforwardly shown that the average velocity of a SISOW is positive and time independent. There is a unique velocity for each position which does not represent the actual velocity of the quantum particles of the beam at any time because it averages the velocities corresponding to incident and reflected particles. On the contrary, the velocity of a particular wave packet at each position depends on time, $\mathrm{v}_{\mathrm{t}_{\mathrm{o}}}(\mathrm{x}, \mathrm{t})$ and is basically positive when the wave packet is incident and negative when it has already been reflected by the barrier. The main advantage of the SISOW is that it allows computing the average velocity as an average over the velocities $v_{t_{0}}(x, t)$ of the ensemble of wave packets. In this regard, we can obtain a probability distribution of velocities given by:

$$
\begin{aligned}
P_{\Psi}(v, x)=\frac{1}{2 \pi \hbar} \int_{-\infty}^{\infty}\left|\Psi_{t_{o}}(x, t)\right|^{2} . \\
\quad \delta\left(v-v_{t_{o}}(x, t)\right) \cdot d t_{o}
\end{aligned}
$$

As an example, in Figure 1, we have depicted the position-velocity distribution, $P_{\Psi}(v, x)$, of the SISOW associated to a beam of electrons impinging upon a $20 \AA$ thick $0.04 \mathrm{eV}$ height square potential barrier. Gaussian wave packets with a central energy of $0.015 \mathrm{eV}$ and spatial standard deviation of $70 \AA$ have been used to build up the SISOW. The broadening of the velocity distribution near the barrier can be understood as a direct consequence of the current uniformity. Since the probability presence decreases in the pre-barrier oscillations, the velocity distribution has to be broader to preserve the current uniformity. Finally, we want to notice the presence of the tunneling ridge that accounts for the partial transmission through the barrier.

\section{APPLICATIONS}

A generalization of the Landauer-Büttiker approach is proposed by using SISOWs instead of Hamiltonian eigenstates. This generalization can be very useful to study time dependent phenomena in actual mesoscopic devices. In particular, different SISOWs (each one associated to an energetic channel) model the conductance, and the thermal reservoir concepts can be invoked to occupy the global density matrix of the system. The resulting density matrix is also diagonal in the energy 

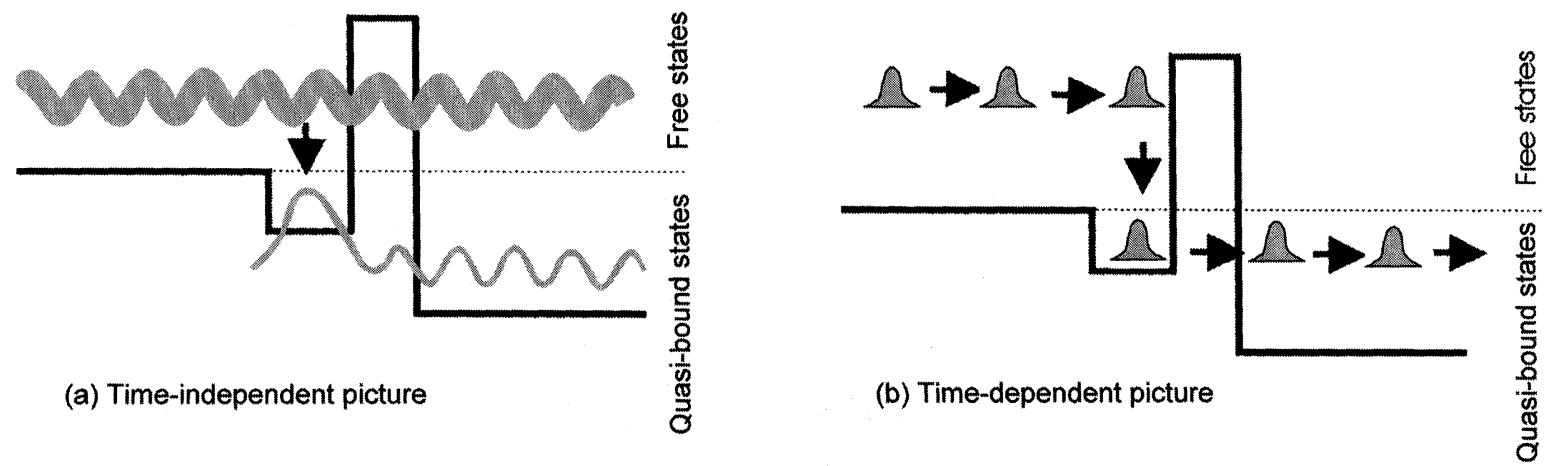

FIGURE 2 Schematic representation of the current carried by the quasi-bound states. Due to the scattering in the well between free-states and quasi-bound states, these states contribute to the total current in heterostructures. (a) Time independent picture using Hamiltonian eigenstates and (b) Time dependent picture using time-dependent wave-packets (SISOW).

representation. Within this approach, we have developed a Quantum Monte Carlo simulator based on Bohm trajectories and wave packets to study actual RTD [4]. These results show the technical viability of the present proposal.

Moreover, another advantage of our proposal is that it can be extended to study non-coherent transport within a simple and versatile framework. In this regard, it has been repeatedly pointed out in the literature that the treatment of dissipative processes in far from equilibrium conditions requires non-diagonal elements in the density matrix [5]. Since the density matrix of a single wave packet is non-diagonal, the presence of nondiagonal elements in the density matrix of the global system can be 'intuitively' described as a process of creation or annihilation (due to different relaxation processes) of the wave packets that compound the SISOWs (see Fig 2b). Notice that creation or annihilation of Hamiltonian eigenstates do not provide non-diagonal elements (see Fig 2a [6]). Several approaches can be found in the literature to rigorously determine the population of these non-diagonal elements in the density matrix [7].

In conclusion, we have presented an exhaustive study of the possibilities of studying electronic transport in tunneling devices in terms of SISOW: Stationary Incoherent Superposition of Wave packets. We point out how this approach provides dynamic information (not contained in the Hamiltonian eigenstates) which can be of great importance to study non-equilibrium carrier dynamics in high speed-semiconductor: current fluctuations, relaxation and transient phenomena in mesoscopic systems.

\section{Acknowledgement}

This work has been partially supported by the DGES under project number PB97-0182.

\section{References}

[1] Landauer, R. (1970). Philos. Mag., 21, 863; Büttiker, M., Irmy, Y., Landauer, R. and Pinhas, S. (1985). Phys. Rev. B, 31, 6207.

[2] Garraway, B. M. and Suominent, K. A. (1995). Rep. Prog. Phys., 58, 365 .

[3] Holland, P. R., The Quantum Theory of motion (Cambridge University press, Cambridge, 1993); Bohm, D. and Hilley, B. J. The undivided Universe (Toutledge, New York, 1993); Oriols, X. et al., Phys. Rev. A, 54, 2594 (1996).

[4] Oriols, X. et al. (1998). Appl. Phys. Lett., 72, 806.

[5] Frensley, W. R. (1990). Rev. of Mod. Phys., 62, 745.

[6] Fischetti, M. V. (1998). J. Appl. Phys., 83, 270.

[7] Rossi, F., di Carlo, A. and Paolo Lugli (1998). Phys. Rev. Lett., 80, 3348. 

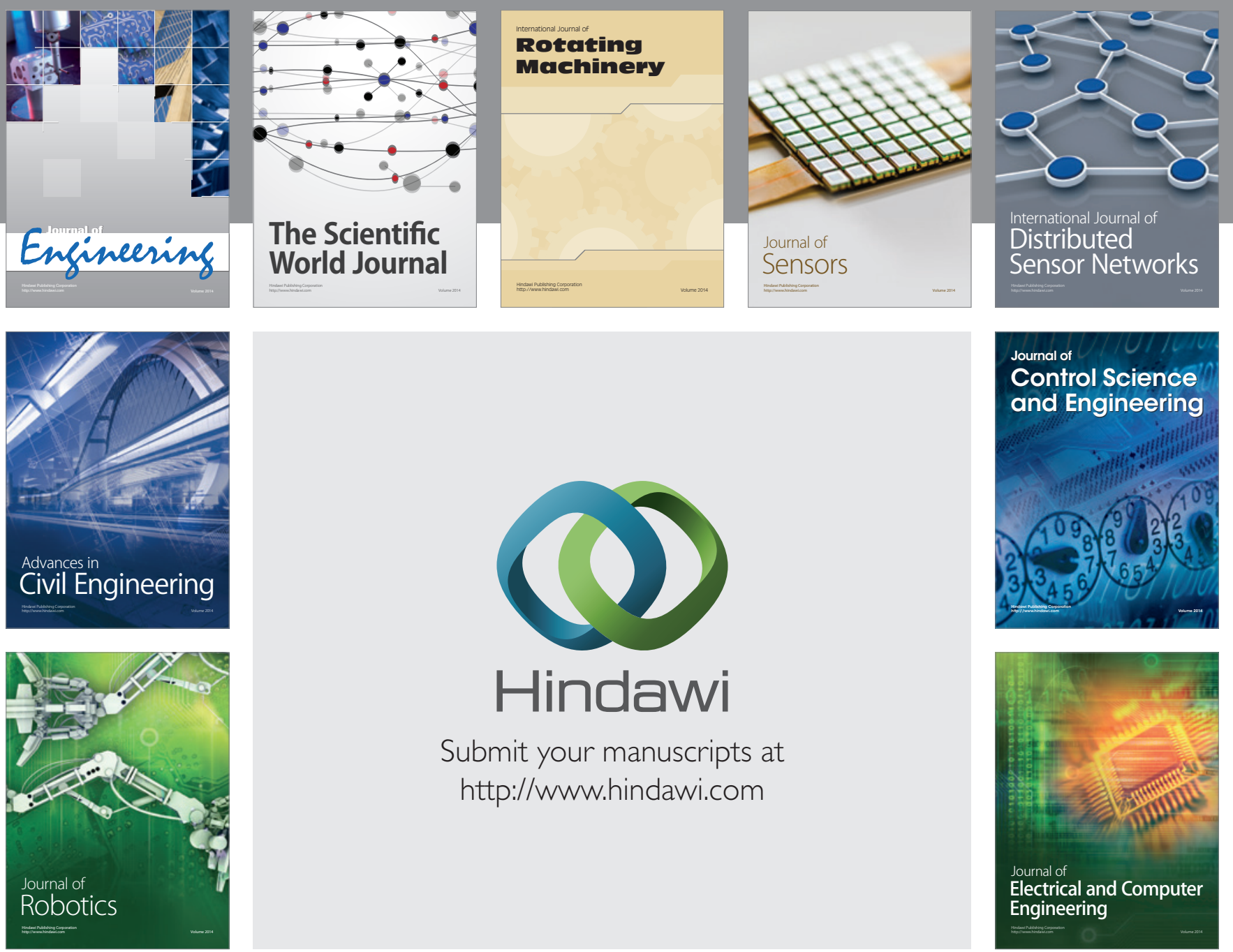

Submit your manuscripts at

http://www.hindawi.com
